\title{
Aufsuchende Soziale Arbeit in Konflikten um städtische Räume
}

\author{
Margarete Killian • Moritz Rinn
}

Eingegangen: 16. Oktober 2019 / Angenommen: 20. April 2020 / Online publiziert: 25. Oktober 2020

(C) Der/die Autor(en) 2020

Zusammenfassung Vor dem Hintergrund der Beobachtung, dass Soziale Arbeit im Kontext neoliberaler Stadtpolitik als Akteurin in Konflikte eingebunden ist, in denen Aneignungs- und Nutzungsweisen öffentlicher städtischer Räume verhandelt werden, stellen wir in diesem Beitrag Ergebnisse eines explorativen Forschungsprojekts vor, in dem wir untersucht haben, wie Fachkräfte aufsuchender Sozialarbeit von stadtpolitischen Akteur*innen in die Bearbeitung solcher Konflikte eingebunden werden und wie sie sich selbst darin positionieren. Ausgangspunkt war die Annahme, dass Akteur*innen stadtbezogener Sozialer Arbeit in Aneignungskonflikten, in die ihre Adressat*innen involviert sind, über einen kontextspezifischen Handlungsspielraum verfügen, in dem Ziele definiert, Interventionsanlässe gewählt, handlungsleitende Problematisierungen hervorgebracht bzw. angeeignet und entsprechende Handlungsstrategien eingesetzt werden. In der empirischen Analyse konkreter Konfliktereignisse und -konstellationen in drei deutschen Großstädten wird ein Spektrum unterschiedlicher sozialarbeiterischer Vorgehensweisen sichtbar, das von parteilicher Intervention und Konfliktorientierung bis zur Übernahme externer Problematisierungen und der aktiven Beteiligung an einer dislocation problematisierter Personen reicht. Diese unterschiedlichen Positionierungen müssen allerdings nicht nur hinsichtlich der jeweiligen normativen (Selbst-)Verpflichtungen (zwischen Parteilichkeit und Allparteilichkeit, Orts- und Klient*innenbezug, Konflikt- und Problemorientierung) analysiert werden, sondern insbesondere in ihren jeweils spezifischen stadträumlichen und stadtpolitischen Konstellationen.

M. Killian, B.A. $(\bowtie) \cdot$ Dr. phil. M. Rinn Institut für Soziale Arbeit und Sozialpolitik, Universität Duisburg-Essen, Universitätsstr. 2, 45141 Essen, Deutschland

E-Mail: margarete.killian@stud.uni-due.de

Dr. phil. M. Rinn

E-Mail: moritz.rinn@uni-due.de 
Schlüsselwörter Aufsuchende Soziale Arbeit · Öffentlicher Raum · Konflikt • Parteilichkeit/Allparteilichkeit · Stadt · Professionelles Selbstverständnis

\title{
Social work in conflicts over the appropriation of urban space
}

\begin{abstract}
Within the context of neoliberal urban development policies, social work has recently become more and more involved as an actor in conflicts over the appropriation and use of public space. Grounding on this observation, in our explorative research project, we analyze the positions assigned to outreach workers by political-administrative actors, and, how outreach workers are positioning themselves in such conflicts. Our starting point is the assumption, that actors of urban social work involved in conflicts fought out by their clients, have different options to choose occasions for intervention, to define the 'real' problems to be solved or conflicts to be settled, and to realize corresponding strategies of action, and, that these options differ according to the specific local and situational contexts. The empirical analysis of concrete conflictual events and constellations in three German cities shows different approaches of social workers ranging from 'partisan' interventions into conflicts to the adoption of external problematizations and active participation in the dislocation of problematized persons. For further research, we suggest analyzing these different positionings not only considering the normative (self-)commitments (e.g. partisanship or multipartiality; orientation towards 'social conflicts' or 'social problems') but especially within their locally specific spatial and political constellations of power.
\end{abstract}

Keywords Outreach social work · Public space · Conflict $\cdot$ Partisanship/ Multipartiality $\cdot$ City $\cdot$ Professional self-concept

\section{Problemaufriss}

Soziale Arbeit ist seit ihrer Entstehung auf „,soziale Probleme“ der Armut und Marginalisierung in Städten bezogen, und ihre Handlungsrationalitäten und Selbstverständnisse korrespondieren mit sich verändernden städtischen Konflikten und Konstellationen beteiligter Akteur*innen (z. B. Drilling und Oehler 2016). Auseinandersetzungen um gesellschaftspolitische Zielsetzungen, selbst- und fremdzugeschriebene Rollen, Problematisierungsbezüge und professionelle Haltungen, wie sie das Feld Soziale Arbeit generell kennzeichnen, lassen sich gegenwärtig gerade auch im Bereich aufsuchender Sozialer Arbeit beobachten (vgl. Diebäcker 2019; Dirks et al. 2016a). Das wird deutlich in Konflikten um öffentliche ${ }^{1}$ städtische Räume, die im Zuge neoliberalisierender Stadtentwicklungspolitik seit Anfang der 1990er Jahre in deutschen Städten zu beobachten sind (vgl. Wehrheim 2012). Im Blickpunkt stehen Konfliktkonstellationen um als abweichend und störend kategorisierte Verhaltensweisen bzw. mit diesen identifizierte Personen, welche zum größeren

\footnotetext{
1 Wir meinen hier mit ,öffentlich“ keine politisch-normative oder administrative Kategorie, sondern schlicht weitgehend ,allgemein` zugängliche Räume.
} 
Teil zum Adressat*innenkreis der Soziale-Probleme-Profession gehören. So geht es um die (sichtbaren) innerstädtischen Reproduktionsstrategien armer bzw. marginalisierter Personen, um Obdachlosigkeit, als offen bezeichnete ,Drogenszenen“, kriminalisierte Sexarbeit, ,aggressives“ Betteln oder um Jugendliche, deren Präsenz an repräsentativen Orten als Kriminalitäts- oder Desintegrationsphänomen gelesen wird. Produktiv werden dabei auch strukturelle wie alltägliche Rassismen und ethnisierend-kulturalisierende Zuschreibungen. In solchen Konfliktkonstellationen geht es sowohl um Sichtbarkeiten sozial ungleich und different positionierter Personen als auch darum, ob, wie und von wem städtische Räume als Ressourcen angeeignet werden können, um die eigenen Alltage und Reproduktionsweisen zu organisieren (vgl. Bareis und Cremer-Schäfer 2013).

Aufsuchend tätige Sozialarbeiter*innen sind in zahlreiche solcher Aushandlungsprozesse involviert. Mit Marc Diebäcker (2019) kann aufsuchende Soziale Arbeit grundlegend als ,,personenbezogene und raumrelationale Praxis gefasst [werden], die strategisch eingesetzt wird, um Verhältnisse von Normalität und Abweichung sowie Ein- und Ausschließung zu bearbeiten“ und deren ,sozialstaatlich unterstützenden Funktionen für marginalisierte oder problematisierte Personen oder Gruppen“ (Diebäcker 2019, S. 544) konstitutiv mit ordnungspolitischen Dimensionen verwoben sind. Fachkräfte der Straßensozialarbeit arbeiten insofern (mit unterschiedlichen Schwerpunktsetzungen) orts- und adressat*innenbezogen und dies zumeist in Verbindung mit institutionellen Einrichtungen wie Konsum- oder ,Trinkräumen', Kontaktläden, Beratungsstellen oder Notunterkünften, die auf sozialrechtlich definierte Zielgruppen mit anerkannten Bedarfen orientiert sind (vgl. Diebäcker 2019). Sozialarbeiter*innen sind zudem in kommunale Gremien der Ordnungs-, Sozialund Stadtentwicklungspolitik eingebunden, in denen Aneignungskonflikte um öffentliche Räume verhandelt werden. Ihnen werden von politisch-administrativen Akteur*innen, aber auch der lokalpolitischen Öffentlichkeit spezifische Aufgaben zugewiesen. Gleichzeitig handeln sie auf Grundlage eines professionellen, normativen wie strategischen Arbeitswissens. Diskutiert werden dabei insbesondere die Bedeutung von Sozialraumorientierung, von Differenzen zwischen orts- oder adressat*innenorientierten Programmatiken, zwischen fachlichen Haltungen der Parteilichkeit oder Allparteilichkeit, und nicht zuletzt zwischen Zurückweisung oder Übernahme ordnungspolitischer Aufträge wie etwa einer relocation problematisierter Personen (Dirks et al. 2016a, 2016b). Wenn aufsuchende Sozialarbeiter*innen in stadträumliche Aneignungskonflikte involviert und insofern zu stadtpolitischen Konfliktakteur*innen werden, darf aber nicht von einer Determination der Praxis durch Programmatiken und Fachkonzepte ausgegangen werden. Anzunehmen ist vielmehr ein kontextspezifischer und situativer Handlungsspielraum, in dem mit anderen beteiligten Akteur*innen interagierende Sozialarbeiter*innen handlungsleitende Problematisierungen hervorbringen bzw. aneignen, Ziele definieren, Interventionsanlässe wählen und Handlungsstrategien einsetzen.

Diese Überlegungen waren Ausgangspunkt für ein exploratives Forschungsprojekt, in dem wir der Frage nachgegangen sind, erstens auf welche Weise Soziale Arbeit durch politisch-administrative Akteur*innen in die Bearbeitung von Konflikten um die Aneignung städtischer Räume eingebunden wird und welche Aufgaben ihr dabei zugewiesen werden, und zweitens wie sich Akteur*innen Sozialer Arbeit 
selbst zu diesen Konflikten bzw. innerhalb der Arrangements zu deren Bearbeitung positionieren, wie sie eigene Aufgaben definieren, welche Problematisierungen und Handlungsstrategien in Bezug auf ihre Adressat*innen dabei wirkmächtig werden und wie sie mit anderen beteiligten Akteur*innen interagieren. Diese Fragestellung schließt an Forschungen zu „Praktiken der Raumherstellung aufsuchender Sozialarbeit" (Dirks et al. 2016a) an, fokussiert jedoch weniger auf die alltägliche sozialpädagogische Arbeit der Fachkräfte sondern methodologisch auf konflikthafte Situationen, in denen die Aneignung öffentlicher Räume ausgehandelt wird, und insofern auf als relational, situativ und interaktiv begriffene Konfliktkonstellationen. Soziale Arbeit interessiert uns dabei aus einer stadtsoziologischen Perspektive in erster Linie als Akteurin eines städtischen Machtfeldes, das sich zwischen den an Aushandlungen der Aneignung öffentlicher Räume konkret beteiligten Akteur*innen entfaltet (vgl. Rinn 2018, S. 15). Solche Konfliktsituationen und -konstellationen zu untersuchen, kann zu einer empirisch fundierten Analyse der Wissens- und Praxisrepertoires aufsuchender Sozialer Arbeit in städtischen Räumen und der widerspruchsvollen normativen wie strategischen stadtpolitischen Positionierungen von Fachkräften beitragen - und insofern zur von Marc Diebäcker (2019) geforderten ,reaktualisierende[n] Auseinandersetzung mit aufsuchender Sozialer Arbeit" (Diebäcker 2019, S. 540). Wir haben deshalb in einer als Vorstudie für weitere Forschungen konzipierten Untersuchung, konfliktorientierte“ Expert*inneninterviews mit Fachkräften aufsuchender Sozialer Arbeit in fünf deutschen Großstädten durchgeführt, in denen politisch-administrative oder polizeiliche Akteur*innen den Zugriff auf problematisierte Personengruppen bzw. innerstädtische Orte verschärft hatten. Dabei haben wir ähnliche übergeordnete Konfliktkonstellationen ausgewählt, um mögliche Variationen politisch-administrativer Einbindungsstrategien sowie sozialarbeiterischer Positionierungen und Praktiken zu erheben. ${ }^{2}$

Unsere Ergebnisse bestätigen einerseits bisherige Befunde einer ordnungspolitisch und auf relocation orientierten Praxis solcher Sozialarbeiter*innen, die ihren Arbeitsauftrag eher ortsbezogen definieren, und einer tendenziell parteilichen Positionierung von programmatisch adressat*innenorientiert arbeitenden Fachkräften (vgl. Dirks et al. 2016a). Gleichzeitig wird aber deutlich, dass auf dem stadtpolitischen Machtfeld insbesondere a) der selbstgewählte oder zugewiesene Charakter von Interventionsanlässen und b) die ,Problematisierungsarbeit' entscheidende Dimensionen der normativen und strategischen Positionierung aufsuchender Sozialarbeiter*innen ausmachen. In den Blick kommt so eine Bandbreite unterschiedlicher Rationalitäten und Praktiken, die sich teilweise den bisher geläufigen und auch dichotomisierenden Kategorisierungen entziehen bzw. diese überschreiten: Parteiliche Soziale Arbeit agiert etwa strategisch intermediär und ortsbezogene Arbeit an relocation bewegt sich in einem Spektrum zwischen pragmatischer auch parteilicher Arbeit, um Vertreibung zu verhindern, und ordnungspolitischen Rationalitäten, die kaum noch Bezüge zu einer sozialarbeiterischen Fachlichkeit aufweisen, aber gleichzeitig durchaus neue Infrastrukturen und selbstverwaltete Freiräume für die-

\footnotetext{
2 Das Forschungsprojekt „Positionen Sozialer Arbeit in der stadtpolitischen Bearbeitung von Konflikten um den öffentlichen Raum“ (9/2017-2/2018) unter Leitung von Jan Wehrheim war an der Universität Duisburg-Essen angesiedelt.
} 
jenigen erschließen, die sie zu ihren Adressat*innen zählen. Schließlich kommt c) der Arbeit an den stadtpolitischen Kräfteverhältnissen durch stadtpolitisch-öffentliche Intervention eine erweiterte Bedeutung insbesondere der sich parteilich positionierenden Akteur*innen zu. Gerade die Problematisierungsarbeit und die Arbeit an Kräfteverhältnissen verweisen auf eine strategische Position Sozialer Arbeit im stadtpolitischen Machtfeld, die es, so unser Plädoyer, in der empirischen Forschung zu aufsuchender Sozialer Arbeit weiterzuverfolgen gilt.

Im Folgenden skizzieren wir zunächst gegenwärtige stadtpolitische Kontextbedingungen stadtbezogener Sozialer Arbeit und geben einen knappen Überblick über debattierte normative und strategische Positionen. Anschließend stellen wir unsere empirischen Befunde dar und diskutieren drei exemplarische Konfliktkonstellationen. Es folgt eine Diskussion der beobachteten Variationen sozialarbeiterischer Positionierungen und Strategien. Abschließend gehen wir auf mögliche Schlussfolgerungen für weiterführende Forschungen zu Konflikten um öffentliche städtische Räume ein, in die Akteur*innen Sozialer Arbeit involviert sind.

\section{Neoliberale Stadtpolitik und die Ausschließung der,Anderen' als Kontextbedingungen stadtbezogener Sozialer Arbeit}

Städtische Ordnungspolitiken, die sich auf die Bearbeitung von ,Störungen “ in öffentlichen Räumen richten, haben sich in den letzten Jahrzehnten kontinuierlich als Teil von neoliberalen Regierungsweisen entwickelt (Wehrheim 2012; Heeg und Rosol 2007). Vor dem Hintergrund einer polarisierten Entwicklung von Städten in Deutschland und staatlicher Austeritätspolitiken ist eine zentrale Dimension neoliberaler Stadtpolitik noch immer eine spezifisch unternehmerische Rationalität (Harvey 1989). Politisch-administrative Akteur*innen setzen im interurbanen Wettbewerb um Unternehmensansiedlungen, steuerzahlende, einkommensstarke und hochqualifizierte ,Leistungsträger', Events und Tourist*innen auf die Optimierung harter wie weicher Standortfaktoren. Die unternehmerische Regierungsrationalität wird tendenziell in allen Politikfeldern produktiv: Lokale Wirtschaftsförderpolitik wird verkoppelt mit aktiver Bevölkerungspolitik, die Städte als Wohnorte für nachgefragte Fachkräfte attraktiv machen will. Die Produktion urbaner Wohn- und Konsumumfelder und entsprechender kultureller Angebote und Infrastrukturen orientiert sich an Arbeitsund Lebensweisen insbesondere der (in sich diversifizierten) Mittelschichten. Die Beseitigung von ,Störpotentialen“ von als abweichend von Mittelschichtsnormen klassifizierten Personengruppen in Wohnquartieren und öffentlichen Räumen spielt dabei eine nicht unbedeutende Rolle.

Städtische Transformationen unter neoliberalisierten Vorzeichen müssen allerdings als konflikthaft konzipiert werden (Mayer 2013). Sie sind konstituiert durch Aneignungskonflikte um städtische Räume und Ressourcen, die mit breiteren, auch trans-nationalen politischen und gesellschaftlichen Transformationen korrespondieren. In solche Konflikte sind sozial ungleich und different positionierte Bewohner*innen auf unterschiedliche Weise involviert. Das wird sichtbar nicht nur an den Kämpfen etwa gegen Gentrifizierung und für ,Wohnraum für alle“ durch städtische soziale Bewegungen (vgl. Rink und Vollmer 2019), sondern auch an den kontinuierli- 
chen transnationalen Migrationsdynamiken, die insbesondere eine städtische Dimension haben: Neu in Städte ziehende Menschen, die politisch-administrativ qua Staatsbürgerschaft oder Aufenthaltsstatus von bürgerschaftlichen Rechten ausgeschlossen werden, müssen sich Zugänge zu alltäglich benötigten Ressourcen erkämpfen und stellen insofern dominante städtische Aneignungsordnungen (alltags-)praktisch in Frage (Hess und Lebuhn 2014). Und so stehen jüngste Konjunkturen kontrollierender, repressiver bzw. auf Verdrängung von ,Problemgruppen“ gerichtete Strategien der Bearbeitung stadtraumbezogener Konflikte in ebendiesem Zusammenhang: Kommunale Sicherheitsverordnungen werden verschärft, lokale Bettel- und Alkoholverbote für öffentliche Räume erlassen, das Kontrollpersonal von Ordnungsämtern aufgestockt und neue Einheiten und Einsatzstrategien geschaffen, flankiert auch von kontrollintensiven Präsenzstrategien der lokalen Polizeien (Hecker 2018; Berg und Wehrheim 2016). Die jüngeren Migrationen aus außereuropäischen Regionen (Hess et al. 2017) werden als spezifisch städtisches Sicherheitsproblem diskutiert, wobei der Diskurs um Drogenkriminalität, um die insbesondere für Frauen als gefährlich markierten (jungen) männlichen Geflüchteten gerade auch lokalpolitisch artikuliert wird (Huke 2019; Perthus und Belina 2017). Ebenfalls verschärft skandalisiert wird Migration aus östlichen EU-Mitgliedsstaaten: Hier stehen Menschen im Fokus, denen ,Sozialtourismus ' unterstellt wird, die mit Kriminalität und deviantem Armutsverhalten in Verbindung gebracht werden. Jenny Künkel (2018) spricht hier von einer umgekehrten Städtekonkurrenz, in der sich Kommunen möglichst unattraktiv und abschreckend positionieren und Armut und Prekarität gerade erzeugen, indem sie bestimmte Personen von sozialen Rechten ausschließen. Die Arbeit an der selektiven Attraktivität städtischer Räume bleibt so eine kontinuierliche stadtpolitische Aufgabe, in die auch kommunale Sozialpolitik eingebunden ist. Sozialpolitik wird zum Element konkurrenzorientierter Stadtpolitik gerade dann, wenn sie als Kohäsionsstrategie konzipiert wird, die soziale Konflikte abmildern und die disruptiven und dysfunktionalen Effekte verräumlichter sozialer Ungleichheiten einhegen soll und gleichzeitig mit an der Legitimierung des Ausschlusses der undeserving poor arbeitet.

\section{Konflikte oder Probleme? Normative und strategische Positionierungen und Selbstverständnisse aufsuchender Sozialer Arbeit}

Diese knappe Skizze macht deutlich, dass die Frage, ob und wie Akteur*innen aufsuchender Sozialer Arbeit in städtische Konfliktbearbeitungsarrangements eingebunden werden, gegenwärtig an Relevanz gewinnt. Entsprechend steht es an, wirksam werdende normative und strategische Positionierungen und Praktiken empirisch in den Blick zu nehmen. Wir schlagen dafür vor, an der Untersuchung konkreter Konfliktsituationen und Akteur*innenkonstellationen anzusetzen und dabei insbesondere zu fragen, ob Praktiken sozialarbeiterischer Fachkräfte darin eine Problemoder Konfliktorientierung aufweisen. Soziale Arbeit wird aus unterschiedlichen theoretischen Traditionen heraus als Konflikt- oder Problembearbeiterin adressiert, was wiederum in einem spezifischen Verhältnis zur Debatte um Parteilichkeit bzw. All- 
parteilichkeit steht. „Soziale Probleme“ sind zunächst klassische Referenzpunkte für die theoretische Bestimmung Sozialer Arbeit (Groenemeyer 2018; Staub-Bernasconi 2012), die aber durchaus kritisch diskutiert werden. Denn durch eine solche Etikettierung gesellschaftlicher Phänomene werden ,gesellschaftliche Konflikte und Widersprüche zwar aufgegriffen, dann aber in Ordnungs- und Konsensthemen umgewandelt" (Stehr und Schimpf 2012, S. 28). Soziale Probleme dienen als Legitimationskategorien für stigmatisierende, kriminalisierende und ausschließende $\mathrm{Zu}$ griffe auf marginalisierte Personen und können beschrieben werden als ,,von Moral durchzogene Konstrukte, die sich mit den gesellschaftlichen Macht-, Herrschaftsund Ungleichheitsstrukturen - vor allem über die Selektivität der Problemdiagnosen und den daran anschließenden Kontrollmaßnahmen - wirkmächtig verb[i]nden“ (Stehr und Schimpf 2012, S. 29). Der Problembegriff transformiert Macht- und Herrschaftsverhältnisse in Fragen von Norm und Abweichung. Johannes Stehr und Roland Anhorn (2018) beschreiben die Konstruktion sozialer Probleme und deren Übersetzung in bearbeitbare Fälle im Anschluss an Nils Christie ,als Enteignungen vom sozialen Konflikt [...], als Enteignungen von den Möglichkeiten, um die eigene gesellschaftliche Positionierung zu verhandeln und die eigenen Interessen gesellschaftlich zur Geltung zu bringen“ (Stehr und Anhorn 2018, S. 3).

Unter den Akteur*innen Sozialer Arbeit sind - neben konzeptuell in Stadtteilentwicklung eingebundenen Projekten - insbesondere Teile der Wohnungslosen-, Drogen- und Jugendhilfe, die aufsuchend oder im Rahmen von Aufenthaltsangeboten arbeiten, auf Konflikte um öffentliche städtische Räume bezogen, denn ihre Adressat*innen sind in diese auf spezifische Weise involviert. In diesen Konflikten werden Aneignungsweisen, die aus der Perspektive der Problematisierenden mit Störungen verbunden sind, und damit normative Raumordnungen und gesellschaftliche Ausschlüsse, verhandelt. Dabei sehen sich die Adressat*innen Sozialer Arbeit Kriminalisierung, Moralisierung und Pathologisierung ausgesetzt, durch welche die beteiligten Akteur*innen bestehende gesellschaftliche Konflikte - die sich auf dominante ReProduktionsnormen und Integrationsanrufungen beziehen - zu sozialen Problemen machen. Über ihre Adressat*innen zur Konfliktakteurin zu werden, kann für Soziale Arbeit bedeuten, die Auseinandersetzungen bzw. Beschwerdeanlässe nicht als Probleme mit definierten Hilfebedarfen und festgeschriebenen Maßnahmenkatalogen zu bearbeiten, sondern in Interaktionen mit anderen Konfliktbeteiligten Engagement für bestimmte Interessen zu artikulieren und die Beschwerdeanlässe als Konflikte offen zu halten. Das kann Möglichkeiten der Verhandlung über die Problematisierungen ergeben, Forderungen in Bezug auf Konzepte und Maßnahmen, oder aber auch die Verweigerung derselben, vielleicht zugunsten einer größeren Sichtbarmachung der unterschiedlichen Interessenlagen und Machtpositionen. Es besteht aber auch die Möglichkeit, dominante Problematisierungen zu übernehmen und als Interventionsanlässe statt sozialer Ausschlüsse ,abweichende Verhaltensweisen' und ,störende Personen(gruppen)` zu setzen, damit einen primär ordnungspolitischen Auftrag anzunehmen und ein so definiertes, Gemeinwohl‘ als Bezugsgröße zu wählen.

In Zusammenhang mit diesen unterschiedlichen Positionierungsmöglichkeiten wird nun auch über die fachlichen Haltungen der (All-)Parteilichkeit diskutiert. Parteilichkeit kann als konfliktorientierte, Allparteilichkeit als konsens- und pro- 
blemlösungsorientierte Perspektive gelesen werden ${ }^{3}$. Empirische Untersuchungen zu Positionierungen aufsuchender Sozialer Arbeit zeigen nun, dass Fachkräfte in ihren Selbstverständnissen und Praktiken zwischen Parteilichkeit und Allparteilichkeit changieren. Sven Huber (2014) berichtet aus Interviews mit Sozialarbeiter*innen der mobilen Jugendarbeit, dass der Begriff der Parteilichkeit primär für die Probleme zur Anwendung komme, die die Jugendlichen hätten, und nicht für diejenigen, die sie machten (Huber 2014, S. 158). Marc Diebäcker (2014) hebt in seiner Untersuchung eines Projekts mobiler Sozialer Arbeit in Wien hervor, dass Allparteilichkeit dort konzeptuell vorgeschrieben gewesen sei, die Sozialarbeiter*innen jedoch sehr unterschiedliche Positionen dazu einnehmen würden. Diese reichten von starker Ablehnung mit dem Argument, dass Allparteilichkeit ,zu Lasten marginalisierter Personen ginge“ über einen Kompromiss wechselnder Parteilichkeit bis zur Unterstützung dieser Haltung (Diebäcker 2014, S. 217). In diesem Zusammenhang sind auch die von Sebastian Dirks und Kolleg*innen (2016a, 2016b) präsentierten Befunde aus Deutschland und der Schweiz und die hervorgehobene Unterscheidung von orts- und klient*innenbezogenen Arbeitsweisen relevant: Die dort diskutierten territorialisierenden sozialräumlichen Ansätze, die ihre Aufträge als Arbeit an problematischen Raumausschnitten definieren, übernehmen ordnungspolitische Zielsetzungen und lehnen parteiliche Positionierungen teils explizit $\mathrm{ab}$ - wobei aber auch ein paternalistischer Adressat*innenbezug hergestellt wird. Die untersuchten Beispiele adressat*innenbezogener aufsuchender Arbeit weisen zwar ebenfalls einen Gebietsbezug auf, die eigenen Interventionen werden aber von den ,Problemen“ der Adressat*innen her konzipiert. Gleichzeitig bringen sie teils ebenfalls raumordnungspolitische Praktiken zum Einsatz (vgl. Dirks et al. 2016b, S. 104 ff.). An diesen Befunden wird deutlich, dass es sich bei der Übernahme oder Ablehnung problemorientierter ordnungspolitischer Aufträge oder (all-)parteilicher Positionierungen um eine normative Frage handelt, die letztlich in den konkreten Aushandlungssituationen, oder zumindest doch von konkreten Sozialarbeiter*innen in den jeweiligen (stadtpolitischen) Kontexten entschieden wird. Und weiter können auch aus einer verbalisierten Zustimmung zur (All-)Parteilichkeit immer noch sehr unterschiedliche Handlungsweisen folgen, die möglicherweise im Widerspruch zum eigenen Selbstverständnis sowie den (artikulierten) Interessen von Adressat*innen stehen. In jedem Fall ist anzunehmen, dass Sozialarbeiter*innen in Konflikten um öffentliche Räume nicht per se gegen Verdrängung und stadtgesellschaftliche Ausschlüsse ihrer Adressat*innen arbeiten. Vielmehr ist von einem breiten Spektrum normativ-strategischer Positionierungen und Praktiken aufsuchender Sozialer Arbeit in Konfliktsituationen auszugehen. Um diese empirisch zu untersuchen, so wollen wir stark machen, müssen sie als eingebettet in Akteur*innenkonstellationen dieser Konflikte begriffen werden. In solchen Konfliktkonstellationen werden die Selbstpo-

\footnotetext{
3 Parteilichkeit geht von der Unmöglichkeit einer neutralen Haltung aus und schreibt sich zu, die Interessen der Adressat*innen infolge einer Mandatierung zu vertreten (Fritsche und Wigger 2016, S. 75; zur Kritik am Mandatsbegriff: Kunstreich 2001; zu Parteilichkeit: Merchel 2000). Im Theoriediskurs zu Hilfe und Kontrolle ist die Position der Parteilichkeit inzwischen weitgehend einer intermediären Position gewichen (Müller 2012, S. 142), die also allparteilich und neutral für sich in Anspruch nimmt, zwischen mind. zwei konfliktbeteiligten Interessen zu vermitteln. Dieser Position liegt die Annahme herrschaftsfreier Diskursstrukturen zugrunde, die dieses neutrale Vermitteln ermöglichen.
} 
sitionierungen und programmatischen Aufträge - also etwa raumordnungspolitische Gebietsbezüge versus Adressat*innenorientierung oder Parteilichkeit versus intermediäre „schlichtende Konfliktarbeit“ (Diebäcker 2019, S. 552) - konkret produktiv und verhandelt.

\section{Die Positionierung aufsuchender Sozialarbeiter*innen in Konflikten um öffentliche städtische Räume - explorative empirische Befunde}

Vor dem Hintergrund dieser Annahmen fragen wir nach den Problematisierungsarbeiten und strategischen Interventionspraktiken aufsuchender Sozialer Arbeit in Interaktionen mit anderen konfliktbeteiligten Akteur*innen spezifischer stadtpolitischer Machtfelder. Wir präsentieren Befunde aus insgesamt vierzehn leitfadengestützten Expert*inneninterviews mit zwanzig Mitarbeiter*innen von zwölf Einrichtungen der (vorwiegend) aufsuchenden Sozialen Arbeit in fünf Großstädten in Deutschland. Sechs Einrichtungen waren im Bereich Wohnungslosenhilfe, vier im Bereich Suchthilfe verortet. Die Zielgruppe von vier Einrichtungen waren Jugendliche oder junge Erwachsene. Die Städte mit Einwohner*innenzahlen zwischen 250.000 und über einer Million sind in fünf verschiedenen Bundesländern verortet. Sie wurden nach Vorrecherchen politisch-administrativer und medialer Dokumente ausgewählt, da dort a) kommunale Ordnungspolitiken intensiviert, b) zugleich neue Sozialarbeitseinrichtungen geschaffen wurden, sich aber c) angesichts ähnlicher Konfliktfälle Unterschiede hinsichtlich der Konfliktbearbeitung vermuten ließen. Politisch-administrative Akteur*innen banden Soziale Arbeit konzeptuell in Arrangements zur Bearbeitung von Konflikten um städtische Räume ein und dies auf durchaus unterschiedliche Weise in unterschiedlichen räumlichen Situationen und Konfliktkonstellationen. Das sozialarbeiterische Repertoire beinhaltete dabei neben klassisch aufsuchender Beratungs-, Vermittlungs- und mediativer Arbeit auch die Schaffung von Sonderräumen für problematisierte Zielgruppen, die ordnungspolitische Maßnahmen flankierten. Der qualitative Zugang orientierte sich auf konkret beschriebene sozialarbeiterische Praktiken in spezifischen Konfliktsituationen bzw. im Kontext übergeordneter Konfliktkonstellationen. Einer kritisch-interaktionistischen Methodologie entsprechend (Bareis und Cremer-Schäfer 2013) haben wir in den Interviews Erzählungen konfliktbehafteter Situationen und interaktiver Praktiken der Fachkräfte erhoben ${ }^{4}$ und in anschließenden Interpretationsdurchgängen als Konfliktkonstellationen rekonstruiert. Die im Folgenden präsentierten drei exemplarischen Fallbeispiele wurden nach dem Prinzip der größtmöglichen Kontrastierung ausgewählt.

\footnotetext{
4 Die Interviews wurden zwischen Oktober 2017 und Februar 2018 geführt, aufgezeichnet und anschließend teiltranskribiert. Auf methodologisch naheliegende ethnographische Vorgehensweisen sowie die Erhebung der Perspektiven und Praktiken der anderen konfliktbeteiligten Akteur*innen (einschließlich der Problematisierten) mussten wir angesichts begrenzter Mittel verzichten.
} 


\subsection{Exemplarische Konfliktkonstellationen}

\subsubsection{Aufsuchende Jugendarbeit im ,Brennpunkt Innenstadt‘ (Stadt A): Vermittlungsarbeit zwischen Problematisierungsumkehr und , sanfter ‘ Disziplinierung}

In Großstadt A ließ sich zum Untersuchungszeitpunkt eine dominante mediale und stadtpolitische Problematisierung von ,Kriminalität “ und ,Unsicherheit “ im Innenstadtgebiet feststellen. Insbesondere ,Dealer', ,unbegleitete minderjährige Flüchtlinge" und ,Trinker' standen im Mittelpunkt einer skandalisierenden Berichterstattung sowie polizeilicher Kontrollpolitiken, wobei Knotenpunkte des Nahverkehrs und ein innerstädtisch zentral gelegener öffentlicher Park polizeirechtlich als ,gefährliche Orte" definiert und dort in regelmäßigen Abständen verdachtsunabhängige Kontrollen durchgeführt wurden. Flankiert wurde dieses Vorgehen durch eine kommunale Ordnungspolitik, die sich der Strategie eines räumlichen Alkoholverbotes bediente und zudem personell aufgestockt wurde. Gleichzeitig wurde der problematisierte Park städtebaulich, landschaftsgärtnerisch und kulturpolitisch bearbeitet. Eine gewisse koordinierende Funktion kam einem kriminalpräventiven Gremium und dort vernetzten politisch-administrativen und polizeilichen Akteur*innen zu. Dabei war es durchaus kommunalpolitisches Ziel, vor allem die jugendbezogene Soziale Arbeit einzubeziehen. So wurde eine neue Anlaufstelle für die aufsuchende Jugendarbeit im Innenstadtbereich geschaffen, die durch kommunale Mittel (ko-)finanziert wurde. Im Rahmen dieser Kooperation blieb allerdings der Einfluss der - sich explizit parteilich positionierenden - Jugendarbeit auf repressive kontrollpolitische Strategien der Polizei gering bis nicht vorhanden (so auch die Aussagen der Fachkräfte).

Die interviewten Sozialarbeiter*innen beschrieben nun als übergreifende Ausgangslage ihrer Arbeit eine ,Verdrängung von den jungen Menschen aus öffentlichem Raum durch Ordnungspolitik“ (Int_A_01, Abs. 34) ${ }^{5}$ und schilderten mehrere Konfliktkonstellationen, in die sie in den vergangenen drei Jahren involviert waren. Eine dieser Konstellationen konstituiert sich um jenen als ,gefährlichen Ort“ kategorisierten Park, der im Sommer ein wichtiger Aufenthaltsort für die jugendliche Zielgruppe sei, die sich in diesem Fall hauptsächlich aus ,vermeintlich nicht-deutschen“ (ebd., Abs. 96) Jugendlichen zusammensetze. Bei den dort regelmäßig stattfindenden Großkontrollen zur Drogenfahndung würden laut Sozialarbeiter*innen (und Pressemeldungen),,wenn überhaupt nur geringe Mengen an Cannabis“ sichergestellt (ebd., Abs. 44). Die Sozialarbeiter*innen böten nun auf dieser Grünfläche regelmäßige sportliche Aktivitäten an - begründet damit, dass sich dort eben ihre Zielgruppe aufhalte, wobei im Interview implizit ein Zusammenhang zwischen Langeweile und problematisier- und kriminalisierbarem Verhalten der Jugendlichen angedeutet wird, der über das Aktivitätsangebot bearbeitetet werden soll. Gerade vor dem Hintergrund, dass Jugendliche ohne ,befriedigende' Freizeitgestaltung, die sich an einem polizeilich definierten ,gefährlichen Ort“ treffen, ,vermeintlich nicht-deutsch“" sind und damit ganz offensichtlich auch zur Zielgruppe polizeilicher Kontrollakteur*innen gehören, lässt sich das sozialarbeiterische Angebot als eine In-

\footnotetext{
5 Interview mit zwei Mitarbeiter*innen der Einrichtung im Oktober 2017.
} 
tervention in die beschriebene Konfliktkonstellation deuten: als Bekräftigung, dass der öffentliche Park ein Ort für Jugendliche sein soll, aber zugleich auch als konfliktpräventiver Ansatz zum Schutz der Zielgruppe vor Sanktionierung - auch durch ,sanfte' kontrollierende bzw. disziplinierende Strategien.

Im Zuge der Präsenz an diesem Ort gerieten die Sozialarbeiter*innen nun einmal auch selbst in eine Personenkontrolle. Mit ihrer Kritik an dieser Situation, die sie danach im Gespräch mit der Polizei und gegenüber Journalist*innen geäußert hätten, bezeichnen sie die polizeiliche Maßnahme als unverhältnismäßig, wobei die Polizei ihre Rolle, Straftaten zu verhindern bzw. zu verfolgen und aufzuklären, auf mehrere Weise überschritten habe: Erstens sei in dem betreffenden Park in der Vergangenheit keine Häufung von Delikten zu verzeichnen gewesen, die ein solch intensives Eingreifen der Polizei hätte rechtfertigen können. Zweitens hätten die Polizist*innen den aufgrund ihrer Kriegs- und Fluchterfahrung möglicherweise traumatisierten Jugendlichen durch ihr martialisches Auftreten ,Angst gemacht“ und diese sogar „beschimpft“ (ebd., Abs. 45f.), und auch damit ihre eigentliche Aufgabe, „Straftaten“ zu verfolgen (ebd., Abs. 67), verfehlt. Diese Definition der polizeilichen Rolle dient zugleich der Abgrenzung des eigenen Auftrags. Eine Bearbeitung des Konflikts wird aus Perspektive der Sozialarbeiter*innen erst durch ,dieses Vermittelnde“ (ebd., Abs. 98) möglich, bei dem es darum geht, die Interessen ihrer Nutzer*innen in den kommunalen Gremien zu vertreten und dabei auch die Kontrollakteur*innen zu bearbeiten - mittels Entskandalisierung und der Etablierung anderer Normalitätserzählungen über die Jugendlichen. Diese fachliche Position zielt letztlich auf eine Kritik an Verdrängung als bloß räumlicher Verlagerung einer ungerechtfertigt als problematisch definierten Gruppe Jugendlicher.

Zentral auffällig ist nun, dass die Sozialarbeiter*innen in Stadt A ihre Interventionsgegenstände aktiv selbst wählen. Das gilt auch für die Bearbeitung einer anderen Konfliktkonstellation rund um eine städtische Kultureinrichtung, die unmittelbar in der Innenstadt gelegen ist. An diesem Konflikt wird außerdem eine der weiteren Strategien der Konfliktbearbeitung deutlich: Die Arbeit mit Beschwerdeführer*innen. Der beschriebene Konflikt dreht sich dabei um die Nutzung des kostenfreien WLANs der Kultureinrichtung durch Jugendliche:

Da war freies WLAN, dort war es warm im Winter und da haben die sich dann getroffen und die Mitarbeiter*innen waren damit überfordert, und da haben wir Weiterbildung gegeben zur Jugendphase, was ist typisch, und wie können sie vielleicht einwirken, positiv, ohne dass immer die Polizei gerufen werden muss. (Int_A_01, Abs. 40)

Diese Vorgehensweise lässt sich auch als Problematisierungsumkehr deuten: Das Problem liegt nun bei den Mitarbeiter*innen der Kultureinrichtung, die nicht kompetent (genug) sind, mit den Jugendlichen adäquat und nicht sanktionierend umzugehen. Geht es bei dieser Strategie darum, ihre Adressat*innen vor repressiver Polizeipraxis (etwa Durchsetzung des Hausrechts, polizeiliches ,für Ordnung sorgen') zu schützen - oder geht es darum, einen stadtgesellschaftlichen Konflikt ohne Rückgriff auf die Polizei als universelle Problembearbeitungsakteurin zu lösen? Bei letzterem hätten die Sozialarbeiter*innen ein quasi allparteiliches (bzw. auf , gute Konfliktlösung ' innerhalb der Stadtgesellschaft gerichtetes) Interesse an der Arbeit 
mit den Beschwerdeführer*innen, deren Konfliktfähigkeit erweitert werden soll. Die Sozialarbeiter*innen würden sich damit genuin als Vermittler*innen sehen und ihre parteiliche Positionierung verlassen (die die Interessen der Adressat*innen vertreten bzw. diese ,empowern“ will). Hier zeichnet sich eine komplexe Strategie im Verhältnis zu Parteilichkeit, Repräsentation und Vermittlung ab.

Die Sozialarbeiter*innen verstehen die Arbeit an Kontrollakteur*innen und Beschwerdeführer*innen durch öffentliche Interventionen (bis hin zu Protestaktionen gegen Verdrängung), Gremientätigkeit und andere Kooperationsformen sowie Vermittlungs- und Bildungsarbeit als konstitutive Elemente ihrer Arbeit im Interesse ihrer Adressat*innen, welche aber dennoch eine regulative, disziplinierende Arbeit am Verhalten der Jugendlichen umfasst. Damit hat es die aufsuchende Jugendarbeit in dieser Stadt geschafft, sich als zentrale Akteurin in der beschriebenen Konfliktkonstellation um Verdrängung respektive ,Kriminalität und Unsicherheit in der Innenstadt ' zu positionieren und zu etablieren. Dabei ist es ihr gelungen, öffentliche finanzielle Unterstützung für eine neue Einrichtung in der Innenstadt zu akquirieren. Angesichts dessen, dass auch das Ordnungsamt eine Aufstockung von Mitarbeiter*innen und die Polizei die Ausweitung rechtlicher Kontrollinstrumentarien erwirken konnte, könnte man von einer Win-Win-Situation und einer insgesamten Intensivierung der ,Problemgruppenbearbeitung ' sprechen. Gleichzeitig wird auch deutlich, dass die durchaus parteiliche und partiell auch konfliktorientierte aufsuchende Jugendarbeit kaum weitere Bündnispartner*innen zur Bearbeitung der übergreifenden Machtkonstellation hat - es fehlt an einer Skandalisierung etwa durch zivilgesellschaftliche Akteur*innen oder auch stadtpolitische Initiativen, die etwa auch die deutlichen Hinweise auf racial profiling seitens der Polizei aufgreifen würden.

\subsubsection{Aufsuchende Arbeit mit der,Trinkerszene“ (Stadt B): Umsiedlung als Kompromiss - Problemlösung als Parteilichkeit?}

In Großstadt B besteht eine langjährige Tradition der ordnungs- wie sozialpolitischen Bearbeitung von ,Problemplätzen', an denen sich drei problematisierte Gruppen in unterschiedlicher Kombination aufhielten: ein ,Drogenmilieu', Obdachlose und Personen, die zwar über eigenen Wohnraum verfügen, aber mit dauerhafter Erwerbslosigkeit und öffentlichem Alkoholkonsum in Verbindung gebracht werden (,Trinker'). Die Plätze sind vor allem in Innenstadtnähe, aber auch in periphereren Stadtteilen verortet und die Stadtverwaltung macht als Beschwerdeführer*innen insbesondere Anwohner*innen, andere Nutzer*innen (etwa von Spielplätzen), Gewerbetreibende und Passant*innen aus, wobei aber auch touristisch relevante Orte im Fokus stehen. Um den Hauptbahnhof wurde ordnungsrechtlich ein temporäres Alkoholverbot erlassen, zudem war die Einführung eines kommunalen Ordnungsdienstes in der Diskussion. Die untersuchte Einrichtung wurde explizit mit dem Auftrag geschaffen, gemeinwesenorientiert im Umfeld problematisierter Orte tätig zu werden und dabei auch präventiv auf den Wohnraumerhalt ihrer Zielgruppe hinzuarbeiten. Mit diesem Auftrag werden ihre Dienste temporär „eingekauft““ 
(Int_B_06, Abs. 7) ${ }^{6}$. Die Arbeitsweise der Einrichtung und die Interventionsanlässe, die der interviewte Sozialarbeiter beschreibt, sind insofern vorwiegend ortsbezogen. Das zeigt sich beispielhaft in einem vom Sozialarbeiter geschilderten Konflikt um einen öffentlichen Platz, der in jüngerer Zeit unter anderem durch die Eröffnung einer Kultureinrichtung stadtentwicklungspolitisch aufgewertet worden sei. Die dort präsente problematisierte Gruppe beschreibt der Sozialarbeiter als ,tief im Stadtteil verwurzelt“, ,die meisten Leute [sind] im Stadtteil geboren“ und würden sich „,von kleinauf" kennen (ebd., Abs. 15). Ein Umzug dieser Gruppe in eine nahegelegene Unterführung sei im Rahmen der baulichen Aufwertung vorgesehen gewesen, habe aber nie stattgefunden, da ,die Leute [...] nach wie vor an den alt angestammten Platz" (ebd., Abs. 17) gegangen seien. Der Sozialarbeiter beschreibt, dass die Personen mit ihren Hunden auf vorbeigehende Schulklassen und Eltern mit Kindern „,bedrohlich“ wirkten. Die „Lösung“ dieser Konstellation, habe nun darin bestanden, dass er bzw. seine Kolleg*innen mit der Kultureinrichtung und der Gruppe folgenden Kompromiss ausgehandelt hätten:

[...] okay, die Bänke [hier] könnt ihr benutzen, diese Bänke unmittelbar am Weg nicht. Bei jedem Besuch haben wir die Leute darauf hingewiesen, haben gesagt: ,ihr nehmt bitte diese Plätze ein“, und nach ein paar Wochen hat sich das dann bei den Leuten auch so eingebrannt. Und dann haben wir halt da auch immer wieder, wenn es mal wieder ein bisschen ins Schleifen gekommen ist, die Leute darauf hingewiesen: ,ihr wisst, es gibt diesen Kompromiss, die [Kultureinrichtung] lässt euch hier, wenn ihr diese euch zugewiesenen Bänke in Beschlag nehmt.“ (ebd., Abs. 11)

Der Sozialarbeiter übernimmt hier selbst Problematisierungen, die auf eine illegitime bzw. problematische Raumaneignung der Gruppe verweisen. Die dargestellte Kommunikation zur Durchsetzung des „Kompromisses“ macht deutlich, dass er hier selbst in vermittelnder Orientierung mit der Macht der Kultureinrichtung arbeitet. Es steht für die betroffenen Personen also weiterhin die Drohung im Raum, sich gar nicht mehr am Platz aufhalten zu dürfen, sollten sie nicht ausschließlich die entfernteren Bänke nutzen. Mit den Mitarbeiter*innen der Kultureinrichtung hätten er und seine Kolleg*innen die Abmachung getroffen, bei Beschwerden immer zuerst die Sozialarbeiter*innen - und nicht direkt die Polizei - zu kontaktieren.

Wenn in den Blick genommen wird, wie hier durch Stadtentwicklungspolitik und Soziale Arbeit eine spezifische Raumordnung verändert bzw. hergestellt werden soll, und welche Aufgabe dabei Sozialarbeiter*innen übernehmen, fällt neben einer Zonierungspraxis (vgl. Dirks et al. 2016a) eine spezifisch ,inklusive“ Kompromissorientierung auf: Die vollständige Vertreibung der Adressat*innen wird durch relocation abgewendet. Dieser „Kompromiss“ wird letztlich mit den bestehenden Machtasymmetrien (auch in Bezug auf die Definition des Ortes) begründet. Gleichzeitig zeigt dieser Umgang mit Beschwerdeführer*innen auch, dass der Sozialarbeiter sich ihnen gegenüber als spezialisierter Professioneller stark macht, den es bei Problemen anzusprechen gilt, bevor die universelle Problemlöserin Polizei eingesetzt wird. Eine Positionierung, die argumentativ auf das Recht der problematisierten Leute verweist,

\footnotetext{
${ }^{6}$ Interview mit einem Mitarbeiter der Einrichtung im November 2017.
} 
sich öffentliche Räume und Infrastrukturen ohne Restriktionen und Sonderbehandlungen anzueignen, findet sich in seiner Erzählung nicht. Dennoch beschreibt er an anderer Stelle als „Grundkonsens“ seines Teams, „,dass die Stadt oder der öffentliche Raum allen gehört, und dass auch unsere Klientel da ein Anrecht drauf hat" (Int_B_06, Abs. 33). Er und seine Kolleg*innen arbeiteten insofern ,parteiisch“, sie würden

versuchen, dass unser Klientel weiterhin diesen Platz oder diesen öffentlichen Raum nutzen kann, aber natürlich wirken [wir] dann auf unsere Leute schon ein oder [...] diskutieren wir schon auch Lösungsvorschläge, wo unser Klientel sich auch einbringen kann, welche Einbußen sie denn hinnehmen würden um weiterhin diesen Platz nutzen zu können. (ebd.)

Insgesamt findet auch hier eine Identifizierung mit einem orts- und zielgruppenbezogenen und dabei hilfe- wie ordnungspolitisch orientierten Auftrag statt. Eine Notwendigkeit, mit Auftraggeber*innen oder auch Beschwerdeführer*innen in eine Auseinandersetzung um Aufenthaltsrechte für ,alle“ einzutreten, besteht offenbar nicht. Dennoch handelt es sich, wenn davon ausgegangen wird, dass Parteilichkeit in erster Linie als Selbstzuschreibung funktioniert, auch um eine begrenzt parteiliche Problemorientierung, und die Selbstbezeichnung als „Kitt der Gesellschaft“ (ebd., Abs. 95) stellt ihn als Vermittler zwischen sozial Ungleichen heraus. Die Potentiale einer Entproblematisierung der Adressat*innen, die der Sozialarbeiter im Interview selbst artikuliert - er legitimiert ihren Aufenthalt etwa darüber, dass es sich um alteingesessene Stadtteilbewohner*innen handele, die deshalb ,dazugehörten', und beschreibt sie kaum als Leute ,mit Problemen “ - schöpft er jedoch nicht aus, vielmehr übernimmt er eine klassische Perspektive der Überführung gesellschaftlicher Konflikte in bearbeitbare Probleme.

\subsection{3 ,Randgruppenbearbeitung' als Umsiedlung und Vertreibung (Stadt C): Nicht- parteiliche Soziale Arbeit?}

Großstadt $\mathrm{C}$ ist durch eine ausdifferenzierte Konfliktlandschaft geprägt. In einigen innenstadtnahen Stadtteilen sowie in den City-Bereichen rund um den Hauptbahnhof werden regelmäßige Konflikte ausgetragen, an denen neben polizeilichen, kommunal-administrativen sowie sozialarbeiterischen Akteur*innen auch unterschiedlich positionierte Anwohner*innen- und Stadtteilinitiativen beteiligt sind: Es geht hier mehr oder weniger kontrovers um ,Umsiedlungen“ von ,Trinkerszenen“, um die Kriminalisierung und Vertreibung von Drogendealer*innen und -user*innen sowie marginalisierte Sexarbeit, aber auch um abweichende Jugendgruppen. Als ordnungspolitische Instrumente kommen insbesondere Präsenz- und Kontrollpolitiken der Polizei an polizeirechtlich definierten ,gefährlichen Orten“ zum Einsatz, aber auch das Ordnungsamt geht etwa gegen problematisierte, vor allem als „osteuropäisch“ kategorisierte Obdachlose vor (so Fachkräfte im Interview).

Im Zentrum der folgenden Analyse steht nun ein zentraler öffentlicher Platz in einem Subzentrum der Stadt, wo im Kontext einer stadtteilentwicklungsstrategischen Diskussion ein Alkoholverbot erlassen wurde. Die dortige ,Trinkerszene" wird seit Anfang der 2010er Jahre sozialarbeiterisch bearbeitet. Nach dem Scheitern eines 
Projektes zur arbeitsmarktpolitischen Reintegration wurde in der Nähe des Platzes eine Tagesaufenthaltsstätte für Menschen ,mit Suchtgefahr' eröffnet, in der leichte alkoholische Getränke konsumiert werden dürfen und auch Sozialberatung stattfindet. Ein Mitarbeiter stellt die Einrichtung im Interview auch als Raum dar, den die Nutzer*innen (teilweise) selbst verwalten könnten (Int_C_13, Abs. 6) ${ }^{7}$. Allerdings trug dieses alternative Aufenthaltsangebot kaum dazu bei, dass sich nicht weiterhin problematisierte Personen den öffentlichen Platz aneigneten. Im Zusammenhang mit einer anhaltenden Berichterstattung lokaler Medien über eine sich zuspitzende Lage informierte der damalige Bürgermeister im Herbst 2016 das Kommunalparlament über Strategien zur „Verbesserung der Aufenthaltsqualität“, wobei ein Alkoholverbot ins Spiel gebracht und ein Jahr später beschlossen wurde. Im entsprechenden Antrag wird die Arbeit der Tagesaufenthaltsstätte durchgehend positiv hervorgehoben, auch wenn die bisherigen Maßnahmen nicht die gewünschten Ergebnisse gezeitigt hätten. Gleichzeitig wird im Interview mit dem Mitarbeiter deutlich, dass dieser sich der medialen und kommunalpolitischen Problemdefinition, dass der Aufenthalt einer, Trinkergruppe " auf dem öffentlichen Platz ausschließend auf andere Personen wirke, vollständig anschließt. Auch zum Alkoholverbot positioniert er sich explizit zustimmend, reklamiert es sogar als seine eigene Idee. Im kommunalpolitischen Gremium habe er auch gegen ,linke“ Kritiker argumentiert, dass durch ein Alkoholverbot keine Verdrängung stattfände, da ja das Aufenthaltsangebot in seiner Einrichtung bestünde. Deutlich artikuliert er dabei auch eine Distanz zur ,klassischen“ (ebd., Abs. 58), als unbedingt parteilich beschriebenen Sozialarbeit. Die Zusammenarbeit mit politisch-administrativen Akteur*innen beschreibt er durchweg positiv und konfliktfrei. Die Einrichtung und das Alkoholverbot stellt er als sich ergänzende Maßnahmen dar, die den problematisierten Leuten kommunizieren sollen: ,wir tun was für euch [...], aber bitte haltet euch nicht mehr an den Plätzen auf" (ebd., Abs. 22). Damit konzipiert der Sozialarbeiter das Alkoholverbot als Aufforderung an die problematisierten Personen, das Angebot der Sozialen Arbeit, welches er als „,softe Maßnahme“ (ebd., Abs. 18) bezeichnet, zu nutzen. Gleichzeitig grenzt er sich jedoch auch explizit davon ab, dass an sein Projekt der ordnungspolitische Anspruch gestellt werde, alle Menschen vom Platz zu holen: Dies bleibe die Aufgabe der kommunalen Ordnungspolitik. Insgesamt fällt auf, dass er mögliche legitime Interessen seiner Adressat*innen in keiner Weise erwähnt, sondern eher defizitorientiert mangelnde Tagesstruktur, Pünktlichkeit, Nüchternheit und Eigenverantwortlichkeit thematisiert.

Die Entstehung und Arbeit der Tagesaufenthaltsstätte ist also unmittelbar Teil der Geschichte der Bearbeitung und Vertreibung von ,Problemgruppen' an einem repräsentativen öffentlichen Ort. Damit ist es einer Akteurin Sozialer Arbeit gelungen, einen städtischen Aneignungskonflikt zur Initiierung eines Projektes zu nutzen, das sich gegenüber den eigenen Adressat*innen explizit nicht-parteilich positioniert. Die Einrichtung ist somit zugleich nur peripher als Bearbeitung der zugeschriebenen Probleme der ,Trinker“ als Adressat*innen Sozialer Arbeit, die zugespitzt als Arbeitsunfähigkeit definiert werden, zu verstehen. Das wird besonders deutlich, wenn der Sozialarbeiter darauf hinweist, dass das Tagesaufenthaltsangebot sich auch

\footnotetext{
${ }^{7}$ Interview mit einem Mitarbeiter der Einrichtung im Februar 2018.
} 
nicht an alle Personen richten könne, die sich am Platz aufhielten. Einerseits gebe es Personen, die partout nicht in die Einrichtung kommen wollten, andererseits das „Problem der Osteuropäer“, assoziiert mit einem „Prekariat“ mit erhöhtem Aggressionspotenzial, mit erhöhtem Konsum hochprozentigen Alkohols und schließlich mit „Obdachlosen-Szenerie“ (ebd., Abs. 10). „Die Osteuropäer“ werden so als Problemgruppe konstruiert, auf die die Einrichtung nicht ausgelegt sei, und ihr stadträumlicher und sozialer Ausschluss wird insofern doppelt legitimiert. Gleichzeitig wird, zumindest dem Selbstverständnis nach, für die ,eigenen Trinker' eine räumliche Infrastruktur bereitgestellt, die diese sich selbstbestimmt aneignen können was sich wiederum als adressat*innenorientiert begreifen lässt, was aber nicht mit einer Entproblematisierung einhergeht.

\subsection{Das sozialarbeiterische Handlungs- und Problematisierungsrepertoire}

Nehmen wir zu den exemplarisch analysierten Konfliktkonstellationen auch die zahlreichen weiteren erhobenen Arrangements hinzu, so ist der erste übergreifende Befund, dass es Sozialarbeiter*innen in nahezu allen untersuchten Städten gelingt, sich als anerkannte Konfliktakteur*innen zu positionieren und zu institutionalisieren. Das Handlungsrepertoire und Selbstverständnis der Sozialarbeiter*innen ist dabei breit ausdifferenziert. Dies zeigt sich auch darin, mit wem bzw. an was sie arbeiten: Es sind nicht nur die problematisierten Adressat*innen, sondern auch bestimmte Beschwerdeführer*innen sowie politisch-administrative Akteur*innen und Institutionen der kommunalen Ordnungs- wie Sozialpolitik sowie der stadtöffentliche mediale Diskurs. In Bezug auf die Problematisierungsarbeiten lassen sich mindestens drei Strategien beobachten: Erstens eine Entproblematisierung als Entskandalisierung und Normalisierung der problematisierten Verhaltensweisen der Adressat*innen; möglich wird so eine Problematisierungsumkehr: $\mathrm{Zu}$ bearbeiten sind nun die Beschwerdeführer*innen, die als Akteur*innen eines Konflikts adressiert werden. Zweitens die Übernahme externer Problembeschreibungen gegenüber den Adressat*innen (etwa jener von Polizei, Gewerbetreibenden, Anwohner*innen oder Stadtverwaltung), was eine Arbeit an den Verhaltensweisen und Aufenthaltsorten der Adressat*innen rational macht. Und drittens die Setzung eigener Problemdefinitionen hinsichtlich der Adressat*innen und der jeweils konkret problematisierten Situationen; dies macht eine Arbeit einerseits an den (zugeschriebenen) individuellen Hilfebedarfen, andererseits an den ausschließenden wohlfahrtsstaatlichen Verhältnissen und Infrastrukturen plausibel.

Vor diesem Hintergrund unterscheidet sich das Handlungsrepertoire der Sozialarbeiter*innen deutlich, wobei sich auch Korrespondenzen zu den institutionellen und kommunalpolitischen Kontexten beobachten lassen. Dabei ist es allerdings nicht so, dass eine schärfere städtische Ordnungs- und Ausschließungspolitik zwangsläufig von einer ordnungspolitisch orientierten Sozialen Arbeit flankiert wird - vielmehr können gerade auch als überzogen begriffene Maßnahmen von Polizei und Stadtverwaltung öffentlichen Widerspruch und klare parteiliche Positionierungen nach sich ziehen (wie etwa in Stadt A). Was die ,Umsiedlung' der Adressat*innen betrifft, fällt auf, dass insbesondere diejenigen Sozialarbeiter*innen aktiv daran mitarbeiten, deren Projekte explizit mit dem Auftrag zur Befriedung spezifischer ,Problemplätze ‘ 
verkoppelt sind, was die Befunde von Sebastian Dirks und Kolleg*innen (2016a) in gewisser Weise bestätigt. Allerdings kann dies im Sinne einer Positionierung erfolgen, die als ,teilweise schon parteilich, aber realistisch' beschrieben werden kann, und die relocation mit einem Schutz der Adressat*innen vor mächtigeren Akteur*innen begründet (wie in Stadt B), oder gewissermaßen konzeptuell, wenn die Problemkonstruktionen der Auftraggeber*innen übernommen werden und so Ordnungs- und Sozialpolitik, Hilfe und Kontrolle tendenziell identisch werden (wie in Stadt C) ${ }^{8}$. Die dabei geschaffenen Sonderräume dienen gewissermaßen der Legitimation des Ausschlusses der Adressat*innen aus den ,normalen“ öffentlichen Räumen (analog zu dem Prozess, den Henning Schmidt-Semisch und Jan Wehrheim (2007) für Drogenkonsumräume und Sebastian Dirks et al. (2016b) für ihr Fallbeispiel ,orstbezogener' Quartiersarbeit beschreiben). Tendenziell kann die NichtNutzung, sanfter" Angebote, als welche bspw. die Sonderräume gedacht sind, zur Legitimation ,harter‘ Eingriffe herangezogen werden: Nehmen ,Problemgruppen im öffentlichen Raum“ sozialarbeiterische Angebote nicht wahr und lassen sich etwa nicht auf ,sozialverträgliche ' Räumungen ihrer Schlafplätze oder ,Umsiedlungen ‘ ihrer Treffpunkte ein, wird damit repressivere soziale Ausschließung legitimiert.

Auch jene Sozialarbeiter*innen, die sich eher parteilich als ordnungspolitisch positionieren, müssen Interaktionen mit ihren Adressat*innen immer vor dem Hintergrund einer solchen Drohkulisse gestalten - und gerade dies macht eine ,sanft kontrollierende" Position pragmatisch plausibel. Die insofern vermittelnde Praxis und implizite Übernahme auch ordnungspolitischer Aufgaben ist also weniger einem programmatischen Defizit geschuldet als einer strategischen Positionierung in konkreten Kräfteverhältnissen. Gleichzeitig ist zu beobachten, dass insbesondere explizit parteiliche Sozialarbeiter*innen stärker an den ordnungs- bzw. sozialpolitischen Akteur*innen und Infrastrukturen und auch an den Beschwerdeführer*innen arbeiten. Hier werden Problemkonstruktionen umgedreht, oder auch mit einer mangelnden Konfliktfähigkeit oder urbanen Kompetenz, Störungen und Konflikte ,auch mal auszuhalten“(Int_A_01, Abs. 98) der Beschwerdeführer*innen argumentiert, die es zu verbessern gelte. Gerade in Bezug auf die Problematisierung von ,Trinkergruppen " geht es aber auch um die Organisation von Kommunikation auf Augenhöhe, also etwa um Gespräche zwischen Beschwerdeführer*innen und Problematisierten, um Kompromisse und Lösungen zu erreichen, und allgemein auch Verständigungsprozesse in Gang zu setzen, die auch zukünftige Konflikteskalationen verhindern sollen. Zentrale beschriebene Strategie ist hier die „Sensibilisierung“ und die Forderung einer wechselseitigen Perspektivübernahme - und zwar auf Seiten beider Konfliktparteien. Hier ließe sich dann übrigens auch argumentieren, dass gerade diese vermittelnde, moderierende, und insofern eher allparteiliche Strategie eine Konfliktaushandlung geradezu ermöglichen soll, indem sie die Problematisierten als Konfliktpartner*innen, also als Personen mit legitimen Interessen auf derselben Ebene wie die Problematisierenden positioniert. Gleichzeitig ist nicht ausgemacht, dass nicht hier auch antagonistische Interessen sichtbar gemacht werden, die sich nicht einfach kompromisshaft (oder konsensorientiert) ,vermitteln', lassen. Was zudem

\footnotetext{
8 Wichtig ist hier auch, dass die Projekte in Stadt B und C sowohl adressat*innen- als auch ortsbezogen agieren, keines aber mit dem Fokus auf Sozialraum oder Quartier.
} 
auffällt: in allen diskutierten Konstellationen agieren die problematisierten Personen bzw. Adressat*innen durchaus widerspenstig und eignen sich die problematisierten Orte beharrlich an - lassen sich also nicht einfach umsiedeln (Stadt B) oder von ihren Treffpunkten durch Alkoholverbote sowie kontinuierliche Polizeieinsätze vertreiben (Stadt A und C). Auch diese fortgesetzten widerspenstigen Aneignungs- und Aushandlungspraktiken, die in unserem Material nur durch Erzählungen der Sozialarbeiter*innen sowie politisch-administrative und mediale Dokumente repräsentiert sind, müssen als Teil des stadtpolitischen Machtfeldes mit eingerechnet werden (und in zukünftigen Forschungen systematisch aus einer Perspektive ,,von unten“ (Bareis und Cremer-Schäfer 2013) mit eingerechnet werden).

Eine weitere zentrale Strategie aufsuchender Sozialarbeiter*innen innerhalb der untersuchten Konfliktkonstellationen ist auch die Mitarbeit in lokalpolitischen Gremien bzw. Kooperationsstrukturen. Hier können ordnungsbehördliche, polizeiliche und wohlfahrtsstaatliche Behandlungen der Adressat*innen kritisiert und möglicherweise auch verändert werden (indem neue Problematisierungen angeboten werden), aber auch Mittel für neue Infrastrukturen eingeworben werden. Nur vereinzelt wird auf die Ebene politisch-öffentlicher Interventionen gewechselt, um in der Stadtgesellschaft auf bestehende Vertreibungskonflikte aufmerksam zu machen: öffentliche Kampagnen (etwa gegen Wohnungsnot) oder Protestaktionen (etwa gegen lokale Alkoholverbote) sind die Ausnahme - sie skalieren die Arbeit an den Kräfteverhältnissen von der lokalen auf die stadtweite Ebene. Und ein weiterer übergreifender Befund dürfte kaum verwundern: In allen Städten werden die jüngeren Migrationsdynamiken zu Themen, mit denen sich die aufsuchenden Sozialarbeiter*innen im Arbeitsalltag auseinandersetzen (müssen), wobei sie hier sehr unterschiedliche Positionen einnehmen. Einige der Fachkräfte kritisieren stadträumliche und sozialstaatliche Ausschlüsse von Bürger*innen der östlichen EU oder auch ordnungspolitische und polizeiliche Kontrollpraktiken gegenüber, jugendlichen unbegleiteten Geflüchteten" und versuchen, dem aktiv entgegenzuwirken (u. a. in Stadt A). Andere Fachkräfte beteiligen sich hingegen aktiv am (stadträumlichen) Ausschluss der „Osteuropäer“, in dem sie etwa in expliziter Abgrenzung ihre ,eigentlichen“ Adressat*innen definieren (wie in Stadt C). Und während nahezu alle interviewten Fachkräfte Vorgehensweisen beschreiben, die als disziplinierend bzw. ,sanft kontrollierend ' begriffen werden können, werden reine Ausschluss-Rationalitäten ausschließlich gegenüber den als fremd definierten Problemgruppen vertreten. Die Bedeutung rassistischer Differenzierungen wird also auch hier deutlich.

Unsere Untersuchungsergebnisse zusammenfassend wird sichtbar, dass das politisch-administrative Interesse an einer stärkeren ordnungspolitischen Einbindung Sozialer Arbeit von aufsuchenden Sozialarbeiter*innen auf sehr unterschiedliche Weise angenommen bzw. genutzt oder auch zurückgewiesen wird. Auf der einen Seite des Spektrums agiert sie als eigensinnige Akteurin in emanzipatorischer Absicht, die soziale Ausschlüsse (bzw. den normativen Anspruch auf gleiche Rechte für alle) thematisierbar macht und Handlungsmöglichkeiten für Leute, die von solchen Ausschlüssen betroffen sind, zu erweitern versucht, ohne dabei aber den normalisierenden und konfliktregulierenden Dimensionen, die sich aus ihren spezifischen Situierungen ergeben, vollends entgehen zu können (Stadt A). Auf der anderen Seite spielen soziale Ausschlüsse als zu problematisierende und zu bearbeitende Bezugs- 
punkte sozialarbeiterischer Praxis keine Rolle und der Ordnungsauftrag ist handlungsleitend, wobei insbesondere rassifizierende Differenzen und damit verbundene sozialstaatliche Ausschließungen affirmiert und mit produziert werden (Stadt C). Zwischen diesen Polen liegt die konsensorientierte Re-Regulierung der Aneignung öffentlicher Räume, die auf eine Art sozialen Frieden zielt (Stadt B).

\section{Schlussfolgerungen und Ausblick}

Beziehen wir die Ergebnisse dieser explorativen Untersuchung nun auf das eingangs skizzierte Spannungsfeld zwischen Konflikt- und Problemorientierung bzw. zwischen Parteilichkeit und Allparteilichkeit, scheint sich auch in unserer Empirie zu bestätigen, dass der klassische ,Rollenkonflikt“ durch eine breitere Anerkennung und „Normalisierung des doppelten Mandats“ (Lutz 2010, S. 268) unproblematisch wird und auch eine ,,an ,Normalzuständen“ orientierte Vermittlerperspektive“ vorzufinden ist, die ausschließende Strukturen aus den Augen verliert (Müller 2012, S. 144). Die Fragen nach den fachlich-politischen Positionierungen aufsuchender Sozialer Arbeit stellen sich nun akut vor dem Hintergrund dessen, dass - so die sich durch unsere Empirie bestätigende These - Soziale Arbeit jüngst vermehrt als Konfliktbearbeitungsakteurin durch politisch-administrative Akteur*innen anerkannt und eingebunden wird, was spezifische Folgen für die Selbstverständnisse und Handlungsstrategien Sozialer Arbeit haben dürfte: $\mathrm{Zu}$ beobachten ist vor allem eine als quasi selbstverständlich angenommene Haltung als ,sanfte Kontrolleure“ (Peters und Cremer-Schäfer 1975), um einen ordnungspolitisch-sanktionierenden Zugriff von Polizei und Stadtverwaltung auf ihr Klientel zu verhindern. Soziale Arbeit wird insofern - auch von politisch-administrativen Akteur*innen - als ein Angebot an die Problematisierten begriffen, das diese annehmen sollten - tun sie dies nicht, ist auch eine sanktionierende Behandlung bis hin zu repressivem Ausschluss legitim. Wenn Soziale Arbeit in dem so aufgespannten Machtfeld alternative Bearbeitungsweisen mit dem Argument stark machen kann, dass Verdrängung keine Lösung, sondern bloß räumliche Verlagerung eines Problems sei, dann muss sie in der Konsequenz eine (disziplinarische) Einhegungsarbeit übernehmen. Sie ist dann an der Herausbildung kleinteiliger städtischer Raumordnungen beteiligt, in denen auch Sonderräume für Problematisierte geschaffen werden. Aus der reinen räumlichen dislocation wird dadurch eine auf den Aufenthaltsort und Verhaltensweisen bezogene relocation (Trinkverbot im öffentlichen Raum, Trinkerlaubnis in der Sozialarbeitseinrichtung). Allerdings, das zeigt das Beispiel aus Stadt C und auch weitere erhobene Konfliktfälle, gilt das vornehmlich für die ,eigenen Problemgruppen', während als ,anders“ Markierte aus der Stadt vertrieben werden sollen.

Nun muss betont werden, dass die empirisch beobachtbaren unterschiedlichen Positionierungen und Praktiken von Akteur*innen Sozialer Arbeit immer in ihren spezifischen stadträumlichen und stadtpolitischen Verortungen analysiert werden müssen. Es macht einen Unterschied, ob sozialarbeiterische Interventionen und Angebote sich auf ein innerstädtisches Konsumzentrum (Stadt A), den repräsentativen Vorzeigeplatz eines Subzentrums (Stadt C) oder auf einen Aufwertungsort im ,Problemquartier“ (Stadt B) beziehen. Und es kann ebenso einen Unterschied machen, 
ob in der stadtgesellschaftlichen Öffentlichkeit Verbündete zu finden sind, mit denen gemeinsam gegen soziale Ausschlüsse interveniert werden kann, oder nicht. Schließlich geht es auch um spezifische Felder des Denk-, Sag- und Machbaren, die sich in der (Zusammen-)Arbeit mit Sozialverwaltung und Stadtteilentwicklungspolitik konstituieren und spezifische Handlungsmöglichkeiten nahelegen, andere verschließen. Wenn nach den produktiv werdenden Handlungsweisen aufsuchender Sozialer Arbeit im Spannungsfeld von Adressat*innenorientierung oder Raumordnungsbezug, Parteilichkeit oder Allparteilichkeit gefragt wird, bedarf es insofern einer integrierten stadtpolitischen Analyse, um Sozialarbeiter*innen als Akteur*innen in Konflikten um die Aneignung städtischer Räume angemessen in den Blick zu bekommen.

Fruchtbar erscheint uns insbesondere ein Fokus auf die Problematisierungsarbeit und die Arbeit an den stadtpolitischen Kräfteverhältnissen, die eng aufeinander bezogen sowohl situativ mit konkreten Beschwerdeführer*innen als auch durch stadtpolitisch-öffentliche Intervention (und insofern als re-scaling) erfolgen. Dies weist auf zwei wichtige Momente hin: Erstens wird durch eine solche Perspektive die (teils eher implizite) Kritik an einer ordnungspolitischen Ortsorientierung aufsuchender Sozialer Arbeit bei Sebastian Dirks et al. (2016b) praktisch erweitert. Diese thematisiert, Adressat*innen würden nicht als „Akteur_innen [verstanden], die ein Problem haben, sondern sie werden zum Problem gemacht, solange sie an bestimmten öffentlichen Plätzen sichtbar sind“ (Dirks et al. 2016b, S. 128). Demgegenüber lässt sich in den Problematisierungsarbeiten der aufsuchenden Sozialarbeiter*innen eine reflexive Befragung der ,Probleme der Adressat*innen“ beobachten: Eine Re-Definition, dass deren Problem zunächst einmal darin bestehe, dass sie von anderen problematisiert werden, ermöglicht eine veränderte Problematisierungsarbeit und damit Strategien, die die Problematisierenden (und insofern die potentiell Ausschließenden) zum Gegenstand sozialarbeiterischer Intervention werden lassen. Gerade wenn es aber um die Verteidigung und Erweiterung von Aneignungsmöglichkeiten der Adressat*innen geht, müsste diese Praxis reflexiv-analytisch stärker ins Zentrum der normativen wie strategischen Überlegungen sozialarbeiterischer Akteur*innen rücken, wie dies etwa in Stadt A (aber auch weiteren erhobenen Konfliktkonstellationen) sichtbar geworden ist.

Diese Arbeit mit den Problematisierenden kann insofern als konfliktpräventiv erscheinen, als dass eine repressive Zuspitzung der Problematisierungsdynamik verhindert werden soll. Sie ist zugleich aber auch konfliktorientiert in dem Sinne, dass hier Interessengegensätze und unterschiedliche Perspektiven sichtbar gemacht und ausgehandelt werden. Dieser Doppelcharakter verweist auf fortbestehend reproduzierte asymmetrische Machtbeziehungen, die auch durch sozialarbeiterische Interventionen nicht einfach beseitigt werden können - und ganz grundsätzlich auch darauf, dass die beobachtbaren Praktiken und Handlungsspielräume von Sozialarbeiter*innen in Konflikten um öffentliche Räume nicht einfach aus ihren jeweiligen administrativen Aufträgen und fachlich-programmatischen Ansätzen abgeleitet werden können. Die dargestellten Konfliktkonstellationen konstituieren sich vielmehr als Teil eines stadtpolitischen Machtfeldes, sodass es eben auch auf die dortigen Kräfteverhältnisse ankommt, d.h. konkret aus der Perspektive sozialarbeiterischer Fachkräfte auf Bündnispartner*innen, die gegen räumliche Ausschlüsse und Kontrollpraktiken gegenüber den ,Anderen“ der bürgerlichen Stadt intervenieren und 
machtvoll Diskurse und Praktiken verändern können. Fehlt es an diesen, liegt eine Mit-Arbeit an dislocation mit dem Ziel, ,Schlimmeres zu verhindern“, als Handlungsoption (auch parteilicher) Sozialer Arbeit nahe. Zentral und für zukünftige Forschungen sollte es also darum gehen, Soziale Arbeit konsequent als relational und situativ agierende Akteurin in einem städtischen Machtfeld mit spezifischen Kräfteverhältnissen zu begreifen, welches sie zugleich mitgestaltet.

Funding Open Access funding enabled and organized by Projekt DEAL.

Open Access Dieser Artikel wird unter der Creative Commons Namensnennung 4.0 International Lizenz veröffentlicht, welche die Nutzung, Vervielfältigung, Bearbeitung, Verbreitung und Wiedergabe in jeglichem Medium und Format erlaubt, sofern Sie den/die ursprünglichen Autor(en) und die Quelle ordnungsgemäß nennen, einen Link zur Creative Commons Lizenz beifügen und angeben, ob Änderungen vorgenommen wurden.

Die in diesem Artikel enthaltenen Bilder und sonstiges Drittmaterial unterliegen ebenfalls der genannten Creative Commons Lizenz, sofern sich aus der Abbildungslegende nichts anderes ergibt. Sofern das betreffende Material nicht unter der genannten Creative Commons Lizenz steht und die betreffende Handlung nicht nach gesetzlichen Vorschriften erlaubt ist, ist für die oben aufgeführten Weiterverwendungen des Materials die Einwilligung des jeweiligen Rechteinhabers einzuholen.

Weitere Details zur Lizenz entnehmen Sie bitte der Lizenzinformation auf http://creativecommons.org/ licenses/by/4.0/deed.de.

\section{Literatur}

Bareis, E., \& Cremer-Schäfer, H. (2013). Empirische Alltagsforschung als Kritik: Grundlagen der Forschungsperspektive der ,Wohlfahrtsproduktion von unten“. In G. Graßhoff (Hrsg.), Adressaten, Nutzer, Agency (S. 139-159). Wiesbaden: Springer VS.

Berg, S., \& Wehrheim, J. (2016). Putzen für Bier. Zur Ambivalenz Sozialer Arbeit. Sozial Extra, 40(6), 6-10.

Diebäcker, M. (2014). Soziale Arbeit als staatliche Praxis im städtischen Raum. Wiesbaden: Springer VS.

Diebäcker, M. (2019). Aufsuchende Soziale Arbeit. In F. Kessl \& C. Reutlinger (Hrsg.), Handbuch Sozialraum (2. Aufl. S. 539-556). Wiesbaden: Springer VS.

Dirks, S., Fritsche, C., Lippelt, M., \& Reutlinger, C. (2016a). Zur pädagogischen Herstellung städtischer Räume zwischen Ort und Klient*in. Empirische Einblicke und theoretische Rückschlüsse. Zeitschrift für Pädagogik, 62(1), 20-33.

Dirks, S., Kessl, F., Lippelt, M., \& Wienand, C. (2016b). Urbane Raum(re)produktion - Soziale Arbeit macht Stadt. Münster: Westfälisches Dampfboot.

Drilling, M., \& Oehler, P. (Hrsg.). (2016). Soziale Arbeit und Stadtentwicklung. Forschungsperspektiven, Handlungsfelder, Herausforderungen (2. Aufl.). Wiesbaden: Springer VS.

Fritsche, C., \& Wigger, A. (2016). Soziale Arbeit und Stadtentwicklung aus reflexiv räumlicher Perspektive. In M. Drilling (Hrsg.), Soziale Arbeit und Stadtentwicklung (S. 71-85). Wiesbaden: Springer VS.

Groenemeyer, A. (2018). Soziale Probleme. In H.-U. Otto, H. Thiersch, R. Treptow \& H. Ziegler (Hrsg.), Handbuch Soziale Arbeit. Grundlagen der Sozialarbeit und Sozialpädagogik (Bd. 6, S. 1492-1507). München: Ernst Reinhardt.

Harvey, D. (1989). From Managerialism to Entrepreneurialism. The Transformation in Urban Governance in Late Capitalism. Geografiska Annaler. Series B, Human Geography, 71(1), 3-17.

Hecker, W. (2018). Umstrittener öffentlicher Raum: Zur neueren Rechtsentwicklung. CILIP, 115, 34-42.

Heeg, S., \& Rosol, M. (2007). Neoliberale Stadtpolitik im globalen Kontext. Ein Überblick. PROKLA. Zeitschrift für kritische Sozialwissenschaft, 149, 492-509.

Hess, S., \& Lebuhn, H. (2014). Politiken der Bürgerschaft. Zur Forschungsdebatte um Migration, Stadt und citizenship. sub\urban. zeitschrift für kritische stadtforschung, 2(3), 11-34. 
Hess, S., Kasparek, B., Kron, S., Rodatz, M., Schwertl, M., \& Sontowski, S. (Hrsg.). (2017). Der lange Sommer der Migration. Grenzregime III (2. Aufl.). Berlin: Assoziation A.

Huber, S. (2014). Zwischen den Stühlen. Mobile und aufsuchende Jugendarbeit im Spannungsfeld von Aneignung und Ordnungspolitik. Wiesbaden: Springer VS.

Huke, N. (2019). Die neue Angst vorm schwarzen Mann. sub urban. zeitschrift für kritische stadtforschung, 7(1/2), 69-92.

Künkel, J. (2018). Die städtische Produktion von „Armutsmigration“. PROKLA. Zeitschrift für kritische Sozialwissenschaft, 191, 283-298.

Kunstreich, T. (2001). Vom Missverständnis eines politischen Mandats Sozialer Arbeit. In R. Merten (Hrsg.), Hat Soziale Arbeit ein politisches Mandat? (S. 121-130). Wiesbaden: VS.

Lutz, T. (2010). Soziale Arbeit im Kontrolldiskurs. Wiesbaden: VS.

Mayer, M. (2013). Urbane soziale Bewegungen in der neoliberalisierenden Stadt. sub urban. zeitschrift für kritische stadtforschung, 1(1), 155-168.

Merchel, J. (2000). Parteilichkeit: ein problematisches Prinzip für professionelles Handeln in der Sozialen Arbeit. In J. Merchel \& L. Hartwig (Hrsg.), Parteilichkeit in der Sozialen Arbeit (S. 49-68). Münster: Waxmann.

Müller, F. (2012). Von der Kritik der Hilfe zur „Hilfreichen Kontrolle“. Der Mythos von Hilfe und Kontrolle zwischen Parteilichkeit und Legitimation. In R. Anhorn, F. Bettinger, C. Horlacher \& K. Rathgeb (Hrsg.), Kritik der Sozialen Arbeit - kritische Soziale Arbeit (S. 123-146). Wiesbaden: VS.

Perthus, S., \& Belina, B. (2017). Policing the Crisis in Bautzen. Soziale Probleme, 28(2), 241-259.

Peters, H., \& Cremer-Schäfer, H. (1975). Die sanften Kontrolleure. Wie Sozialarbeiter mit Devianten umgehen. Stuttgart: Enke.

Rink, D., \& Vollmer, L. (2019). Mietenwahnsinn stoppen. Forschungsjournal Soziale Bewegungen, 32(3), 337-349.

Rinn, M. (2018). Ein Urbanismus der Ungleichheit. sub urban. zeitschrift für kritische stadtforschung, $6(1), 9-28$.

Schmidt-Semisch, H., \& Wehrheim, J. (2007). Exkludierende Toleranz oder: Der halbierte Erfolg der ,,akzeptierenden Drogenarbeit“. Widersprüche, 27(103), 73-91.

Staub-Bernasconi, S. (2012). Soziale Arbeit und soziale Probleme. In W. Thole (Hrsg.), Grundriss Soziale Arbeit. Ein einführendes Handbuch (4. Aufl. S. 267-282). Wiesbaden: VS.

Stehr, J., \& Anhorn, R. (2018). Konflikt als Verhältnis - Konflikt als Verhalten - Konflikt als Widerstand. Widersprüche der Gestaltung Sozialer Arbeit zwischen Alltag und Institution. In J. Stehr, R. Anhorn \& K. Rathgeb (Hrsg.), Konflikt als Verhältnis - Konflikt als Verhalten - Konflikt als Widerstand. Widersprüche der Gestaltung Sozialer Arbeit zwischen Alltag und Institution (S. 1-40). Wiesbaden: Springer VS.

Stehr, J., \& Schimpf, E. (2012). Ausschlussdimensionen der Sozialen-Probleme-Perspektive in der Sozialen Arbeit. In E. Schimpf \& J. Stehr (Hrsg.), Kritisches Forschen in der Sozialen Arbeit. Gegenstandsbereiche - Kontextbedingungen - Positionierungen - Perspektiven (S. 27-42). Wiesbaden: VS.

Wehrheim, J. (2012). Die überwachte Stadt - Sicherheit, Segregation und Ausgrenzung (3. Aufl.). Opladen: Barbara Budrich. 